\title{
Effects of Dislocations on the Oxygen Ionic Conduction in Yttria Stabilized Zirconia
}

\author{
Kazuya Otsuka ${ }^{1, *}$, Katsuyuki Matsunaga ${ }^{2}$, Atsutomo Nakamura ${ }^{2}$, Seiichiro $\mathrm{Ii}^{2}$, \\ Akihide Kuwabara ${ }^{3}$, Takahisa Yamamoto ${ }^{4}$ and Yuichi Ikuhara ${ }^{2}$ \\ ${ }^{1}$ Department of Material Engineering, School of Engineering, The University of Tokyo, Tokyo 113-8656, Japan \\ ${ }^{2}$ Institute of Engineering Innovation, School of Engineering, The University of Tokyo, Tokyo 113-8656, Japan \\ ${ }^{3}$ Department of Material Science and Engineering, Faculty of Engineering, Kyoto University, Kyoto 606-8501, Japan \\ ${ }^{4}$ Department of Advanced Material Science, Graduate School of Frontier Sciences, The University of Tokyo, Tokyo 113-8656, Japan
}

Ionic conductivities of yttria-stabilized zirconia (YSZ) single crystals deformed at high-temperature were measured by the AC impedance method. A correlation between ionic conductivity and dislocation structures of deformed YSZ single crystals were investigated. Electrical conductivity measurements of the deformed YSZ crystals were performed for two different current directions of [1 10$]$ and [1 111$]$. The [1 10$]$ direction is parallel to edge dislocations introduced by the primary slip system, while the [1 $\overline{1} 1]$ direction is normal to the edge dislocation lines. Transmission electron microscopy observations showed that the dislocations due to the primary slip system of (001)[110] were mainly generated

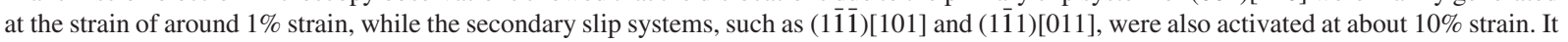
was found that the deformed samples with larger strains exhibited higher electrical conductivities irrespective of the measured current directions. However, the electrical conductivity along [11ㅣ was higher than that along [1ํㅣ, suggesting that mobility of oxygen ion is sensitive to the dislocation structures. From the activation energy for oxygen diffusion in the deformed samples, it was found that the oxygen migration enthalpy for deformed samples became smaller than that for undeformed samples, whereas the association enthalpy for the deformed samples became larger. The increase in the association enthalpy might be due to interaction between oxygen vacancies and dislocations. It is thus considered that oxygen vacancies concentrate around dislocations, and are able to move very quickly along the dislocation lines.

(Received January 9, 2004; Accepted March 25, 2004)

Keywords: dislocations, oxygen ionic conduction, zirconia, single crystal, high-temperature deformation, SOFCs, solid electrolyte

\section{Introduction}

Zirconia $\left(\mathrm{ZrO}_{2}\right)$ has polymorphism of monoclinic, tetragonal and cubic phases. ${ }^{1-3)}$ The cubic phase of $\mathrm{ZrO}_{2}$ with the fluorite structure $\left(\mathrm{c}-\mathrm{ZrO}_{2}\right)$ is stable at high temperature from 1430 to $2650 \mathrm{~K}$, and further addition of metal oxides such as $\mathrm{Y}_{2} \mathrm{O}_{3}, \mathrm{CaO}$ and $\mathrm{MgO}$ can stabilize the cubic phase at lower temperatures. ${ }^{4-10)}$ The doping of such di- or trivalent cations gives rise to formation of oxygen vacancies due to chargeneutrality requirement of the whole systems, ${ }^{11}$ ) and the oxygen vacancies are able to diffuse easily through the lattice. As a result, c- $\mathrm{ZrO}_{2}$ stabilized by the dopants exhibits high ionic conductivities, which is suitable for applications to electrolytes in solid oxide fuel cells (SOFCs). ${ }^{12)}$ However, the ionic conductivity of $\mathrm{c}-\mathrm{ZrO}_{2}$ depends on dopant types and concentrations. ${ }^{13)}$ Moreover, in the polycrystalline materials of $\mathrm{c}-\mathrm{ZrO}_{2}$, which is referred to as fully stabilized $\mathrm{ZrO}_{2}$ (FSZ), the microstructures such as grain size and grain boundary structures are known to affect the ionic conductivity. ${ }^{14,15)}$ For example, Aoki et al. ${ }^{14)}$ pointed out that grain boundaries act as barriers for conduction of oxygen vacancies. This might be due to characteristic atomic structures of individual grain boundaries or segregation of dopants and impurities at grain boundaries. In contrast, Kosacki et al. ${ }^{16)}$ reported that nanocrystalline yttria-stabilized $\mathrm{ZrO}_{2}$ (YSZ) exhibited higher ionic conductivities by a two order of magnitude as compared to the single crystals and polycrystals with larger grain sizes. This result indicates that the grain boundaries might act as rapid diffusion pathways of oxygen ions, ${ }^{17}$ ) because the nanocrystalline specimen contains a larger density of grain boundaries. It can be thus said that there is still a discrepancy

*Graduate Student, The University of Tokyo of a role of grain boundaries for the ionic conductivity of FSZ. However, as is known in a number of metals and ceramics, grain boundaries and surfaces sometimes act as effective diffusion paths, ${ }^{18)}$ such specific defect structures are considered to contribute to significant increase in the oxygen diffusivity in $\mathrm{c}-\mathrm{ZrO}_{2}$.

Similar to grain boundaries, it is known that dislocations can induce rapid diffusion of solutes and vacancies, which is called as pipe diffusion. ${ }^{19-22)}$ In our previous study, ${ }^{23)}$ we paid attention to the effect of dislocations on atomic diffusivity, and studied ionic conductivities of YSZ single crystals deformed at high temperatures. It was found that the deformed YSZ single crystals exhibited higher conductivities with increasing plastic strains. Therefore, it is expected that dislocations in deformed YSZ act as rapid diffusion paths for oxygen vacancies and enhance the electrical conductivity in YSZ. However, it is important to understand the relationship between the electrical conductivity and the dislocation structures, in order to further improve the conductivity of FSZ by introducing dislocations. In this study, YSZ single crystals were deformed by compressive tests at high temperatures, and their electrical conductivities were systematically measured as functions of temperature, current directions and plastic stains. Dislocation structures and distribution were observed by transmission electron microscopy (TEM), and a plausible mechanism of the increased ionic conductivity in deformed YSZ was discussed.

\section{Experiments}

Commercial YSZ single crystals $\left(10 \mathrm{~mol} \% \mathrm{Y}_{2} \mathrm{O}_{3}-\mathrm{ZrO}_{2}\right)$ grown by the skull melting process (Earth Chemical Co. Ltd., Tokyo, Japan) were used as starting materials. YSZ single 


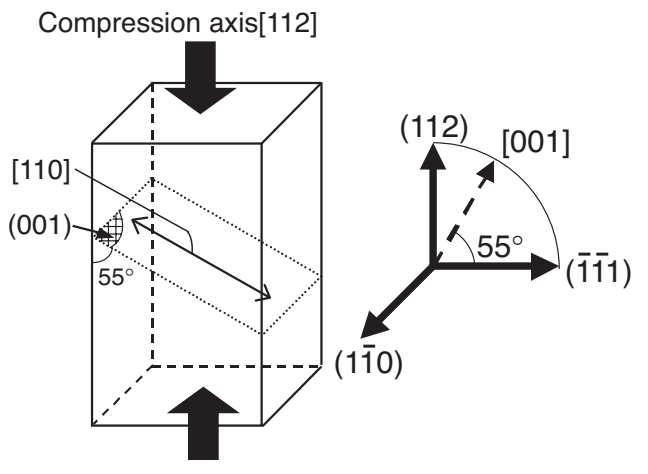

Fig. 1 Schematic illustration of the YSZ specimen for high temperature compression tests.

crystals were mechanically cut in order to obtain specimens for compressive mechanical tests, as shown in Fig. 1. The specimen size was $5.0 \times 5.0 \times 10.0 \mathrm{~mm}^{3}$, and all surfaces of the specimen were mechanically polished to a mirror finish. In the specimen, the [112] direction of the cubic fluorite structure was selected parallel to the compression axis so that the primary slip system of (001)[110] can be preferentially activated due to its high Schmid factor of $0.47 . .^{24,25)}$ Compression tests were conducted in air at $1300^{\circ} \mathrm{C}$ under an initial strain rate of $8.3 \times 10^{-6} \mathrm{~s}^{-1}$, up to the plastic strains of $1 \%$ or $10 \%$.

Electrical conductivities of undeformed and deformed samples were measured by AC impedance measurements. The specimens were uniformly deformed in the central regions, although the edge of the deformed specimen was distorted due to the geometry of single crystal. Thus, samples for $\mathrm{AC}$ impedance measurements were obtained from the homogeneously deformed parts of the single crystals by mechanical cutting. The sample size for AC impedance measurements was about $2.5 \times 2.5 \times 5.0 \mathrm{~mm}^{3}$. In order to remove the influence of the shape change on the electrical conductivities by plastic deformation, the shape of samples was adjusted by mechanical polishing, so that the shape becomes a rectangular parallelepiped. Subsequently, the size of samples for the AC impedance measurement was measured using the micrometer (the accuracy of the micrometer was in the order of $1 \mu \mathrm{m})$.

For impedance measurements, platinum electrodes were applied on the (1히) plane or ( $\overline{1} \overline{1} 1)$ plane of the YSZ samples, and were sintered at $930^{\circ} \mathrm{C}$ for $15 \mathrm{~min}$. Impedance spectra were obtained over a frequency range from $0.1 \mathrm{~Hz}$ to $6.0 \mathrm{MHz}$ at an applied voltage of $1 \mathrm{~V}$ by an impedance analyzer (1260 Impedance/Gain Phase Analyzer, Solartron Analytical, Hampshire, UK). Impedance measurements were conducted for five specimens at each condition in air. Undeformed (0\% deformed) and $10 \%$ deformed samples were measured at temperatures from 250 to $1000^{\circ} \mathrm{C}, 1 \%$ deformed samples were measured at temperatures from 250 to $700^{\circ} \mathrm{C}$ at an interval of about $50^{\circ} \mathrm{C}$. The electrical conductivity at each condition was obtained by averaging measured values for three specimens except for two specimens exhibiting maximum and minimum values. It is noted here that the standard deviation of experimental values for three specimens except for two samples exhibiting maximum and minimum values is about $1 \%$ of the average values. The reproducibility error of the electrical conductivity measurements caused by the uncertainties of thermometer was 3\% at $250^{\circ} \mathrm{C}$, the error may become smaller with increasing temperature (see eq. (1)).

In the present electrical conductivity measurements, two

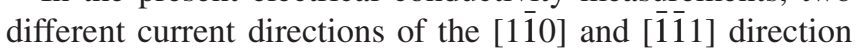
were selected. This is because the compressive tests are expected to introduce a number of dislocations in YSZ crystals due to activation of the primary slip systems, and the dislocations may induce directionality of electrical conductivity, depending on the dislocation structures and distribution. The $[1 \overline{1} 0]$ direction was parallel to the edge dislocation lines expected to be introduced by the compression tests. The [1111] direction is normal to the edge dislocation lines and about $35^{\circ}$ to the screw dislocation lines. For making a comparison with deformed samples, electrical conductivities of undeformed samples were also measured in the similar manner.

Structures and distribution of dislocations in undeformed and deformed samples were observed by TEM. The samples were sliced parallel to the (110) plane or (001) plane; a thickness of the foil is about $0.5 \mathrm{~mm}$. Thin foils for TEM observation were prepared using conventional techniques of mechanical thinning to about $20 \mu \mathrm{m}$ and ion-beam milling to obtain electron transparency. In the case of TEM observations, carbon films were coated on the TEM samples to prevent the samples from charging up. A thickness of the carbon films was about $3 \mathrm{~nm}$. TEM observation was carried out with the JEOL JEM-2010HC system operating at $200 \mathrm{kV}$.

\section{Results}

\subsection{TEM observations}

Figure 2 shows TEM images of $1 \%$ deformed YSZ single crystals. The Burgers vector of almost all dislocations in Figs. 2(a) and (b) was confirmed to be $\boldsymbol{b}=a / 2[110]$ by $\boldsymbol{g} \cdot \boldsymbol{b}$ analysis, which indicates that the primary slip system of (001)[110] mainly acted at the $1 \%$ deformation. In the case of undeformed specimen, dislocations were randomly distributed and the dislocation density was estimated to be as small as about $10^{11} \mathrm{~m}^{-2}$.

In contrast, most of dislocations were observed as black dots in Fig. 2(a). This indicates that dislocation lines in Fig. 2(a) were mostly parallel to the incident beam direction [110]. Figure 2(b) shows the dislocation structures in $1 \%$ deformed samples observed with the incident beam parallel to the [001] direction. It was confirmed from both image that relatively straight dislocation lines with $\boldsymbol{b}=a / 2[110]$ were introduced by plastic deformation. Since the primary slip system of (001)[110] is mainly activated at the $1 \%$ strain, it can be said that many edge dislocations with $\boldsymbol{b}=a / 2[110]$ were generated in $1 \%$ deformed samples. The dislocation density in $1 \%$ deformed samples was estimated to be about $10^{13} \mathrm{~m}^{-2}$, which was about $10^{2}$ times larger than that in undeformed samples.

However, dislocation structures and distribution in the $10 \%$ deformed samples were different from that in the $1 \%$ deformed one. Figure 3 shows the typical bright field images of dislocation structures for the specimen with $10 \%$ defor- 

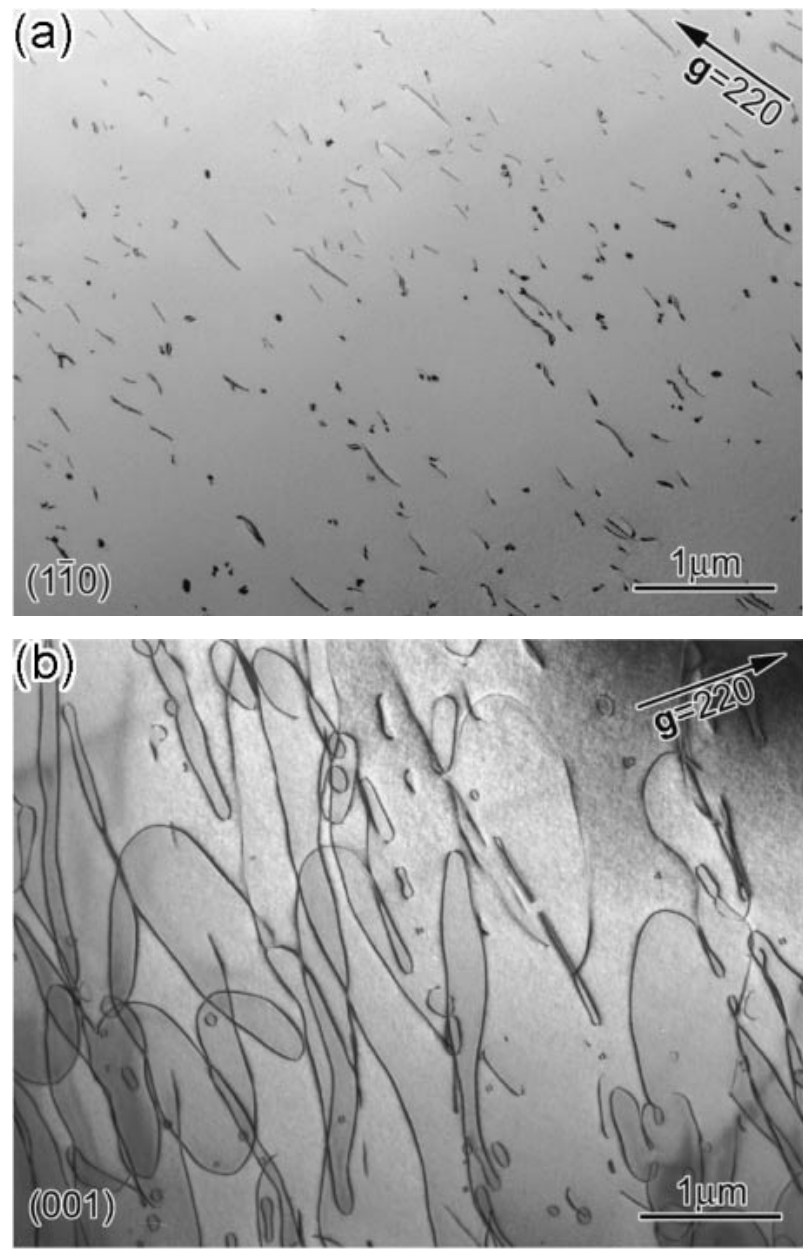

Fig. 2 TEM bright field images of YSZ single crystals deformed at $1 \%$ strain, which were observed (a) parallel and (b) perpendicular to the primary slip plane (001).

mation. In spite of the same incident beam direction of [110] with that in Fig. 2(a), it can be seen that dislocation lines in Fig. 3(a) were more awry and tangled. This is due to the

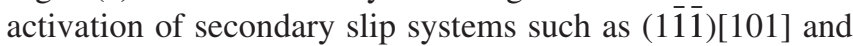
(11) $[011]$ in $10 \%$ deformed samples. ${ }^{26,27)}$

In the AC impedance measurements, temperature of the samples reaches the maximum of $1000^{\circ} \mathrm{C}$. And Pt electrodes of the samples were baked at $930^{\circ} \mathrm{C}$. Dislocation structures in the samples may be changed by these heat treatments. So we observed dislocation structures and distributions in $10 \%$ deformed samples before and after the AC impedance measurements. Figure 3(b) shows the dislocation structures in the $10 \%$ deformed samples after the AC impedance measurements. The dislocation structures and distribution in Fig. 3(b) were almost the same with those in Fig. 3(a). Therefore, it can be said that the heat treatments for YSZ specimens in the present study do not affect the dislocation structures and distributions.

\subsection{AC impedance measurements}

As mentioned in 3.1, dislocation lines in the $1 \%$ deformed samples were relatively straight (see Fig. 2(a) and (b)). In contrast, the dislocation structures in the $10 \%$ deformed samples (see Fig. 3) were more complicated due to the
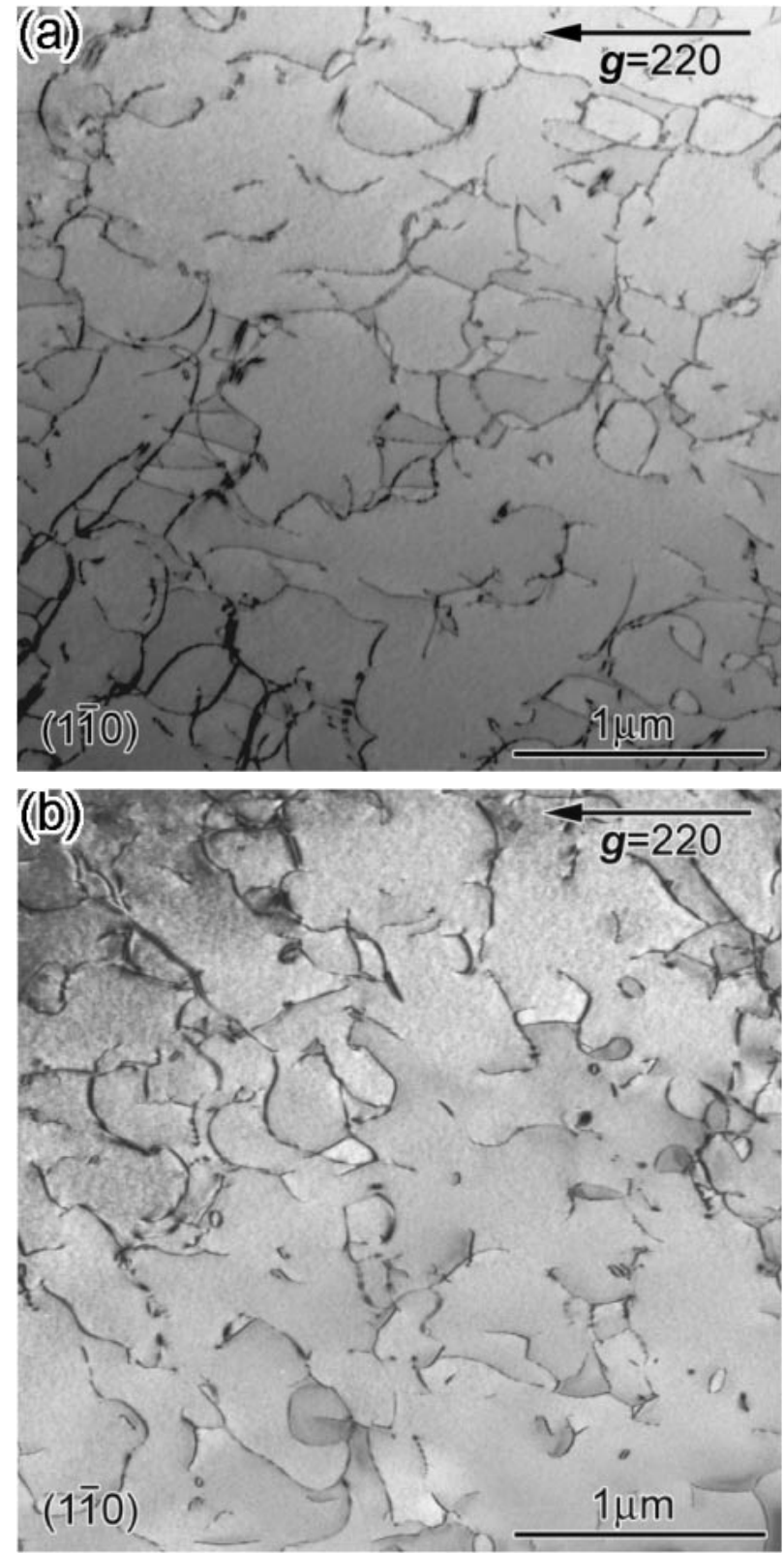

Fig. 3 TEM image of 10\% deformed YSZ single crystals. (a) before and (b) after the thermal treatments. The diffraction vector is $g=220$ and the incident beam is parallel to [1ㅣㅣㄹ.

activation of the secondary slip systems. The electrical conductivity of the deformed and undeformed YSZ single crystals were measured by the AC impedance method, and the effect of the dislocation structures on the ionic conductivity was investigated.

The impedance spectra at $400^{\circ} \mathrm{C}$ for deformed and undeformed samples along [ $\overline{1} \overline{1} 1]$ direction are shown in Fig. 4. Each impedance curve consists of two well-separated arcs, and the diameter of the semicircles corresponds to the electrical resistance for the sample. ${ }^{28,29)}$ It can be seen from Fig. 4 shows that electrical resistance for deformed samples were smaller than that for undeformed samples. We already reported that the electrical conductivity along [1 $1 \overline{1} 0]$ direction for deformed samples was larger than that for undeformed samples. ${ }^{23)}$

Figure 5 shows the measured ionic conductivities of $1 \%$ 


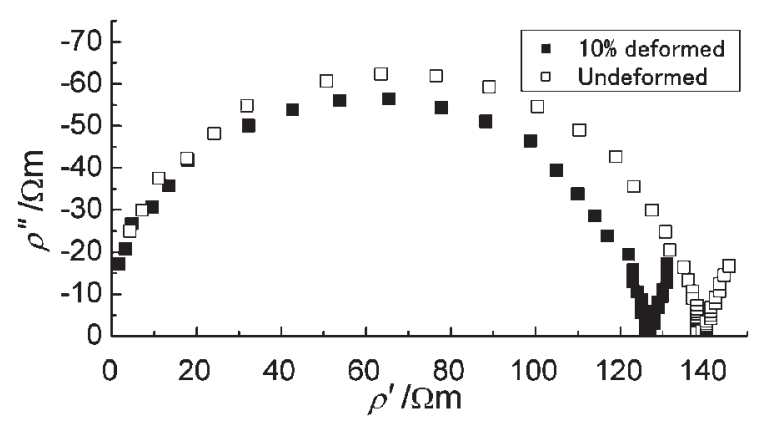

Fig. 4 Complex impedance plots of YSZ single crystals at $400^{\circ} \mathrm{C}$. Filled squares represent $10 \%$ deformed samples. Open squares represent undeformed samples. The current direction is parallel to the [1 111$]$ direction.

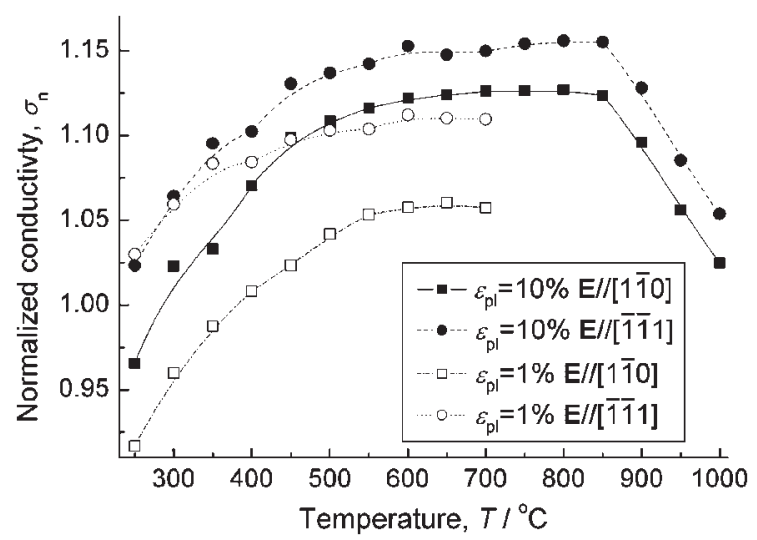

Fig. 5 Temperature dependence of the normalized conducivity $\left(\sigma_{\mathrm{n}}=\sigma_{\text {deformed }} / \sigma_{\text {undeformed }}\right)$ for deformed YSZ single crystals in each strain and each current direction.

and $10 \%$ deformed samples as a function of temperature. In this case, the ionic conductivities of the deformed samples were normalized by the value of undeformed samples obtained at the same temperature conditions, namely normalized conductivity $\left(\sigma_{\mathrm{n}}=\sigma_{\text {deformed }} / \sigma_{\text {undeformed }}\right)$. It can be seen that the $\sigma_{\mathrm{n}}$ values measured along [110] for the deformed samples increase with increasing temperature. At above $400^{\circ} \mathrm{C}$, the $\sigma_{\mathrm{n}}$ values become larger than 1.0 , indicating the enhanced ionic conductivity by the plastic deformation. It should be noted here that the $\sigma_{\mathrm{n}}$ values measured along [1 $\overline{1} 1]$ become much larger than those along [1ํㅣㄹ, and thus the conductivities also depend on the current direction.

The similar trends of $\sigma_{\mathrm{n}}$ can also be found for the $10 \%$ deformed samples. The $\sigma_{\mathrm{n}}$ of the $10 \%$ deformed samples exhibits larger values, as compared to the $1 \%$ deformed case. This indicates that larger plastic deformations result in more increase of the ionic conductivities. However, the $\sigma_{\mathrm{n}}$ values abruptly decrease beyond $850^{\circ} \mathrm{C}$, and approach to 1.0 around $1000^{\circ} \mathrm{C}$.

From the measured ionic conductivities of the undeformed and $10 \%$ deformed samples, activation energies for oxygen ionic conduction were analyzed by the Arrhenius plots shown in Fig. 6. According to the previous studies about ionic conduction in zirconia ceramics, ${ }^{11)}$ oxygen ionic conductivity $(\sigma)$ is given by

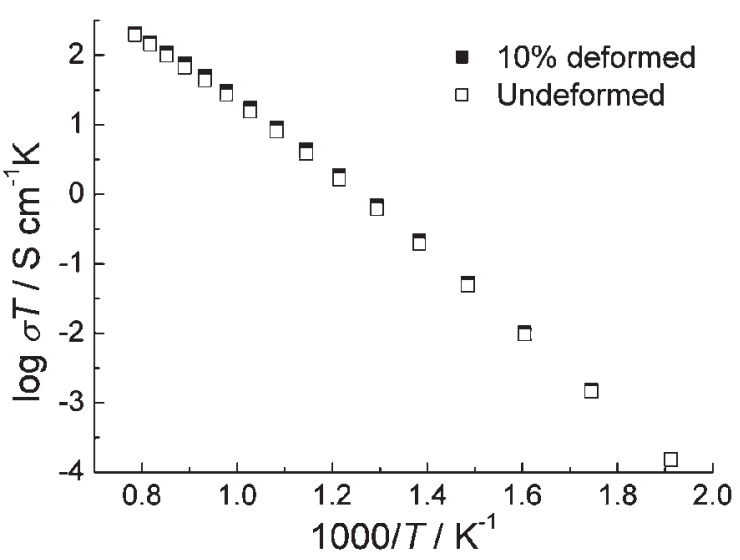

Fig. 6 Arrhenius plots of electrical conductivity along the [1111] direction for deformed and undeformed YSZ single crystals. Filled squares represent $10 \%$ deformed samples. Open squares represent undeformed samples.

$$
\sigma T=A \exp \left(-\frac{E_{a}}{k T}\right),
$$

where $T$ is absolute temperature, $A$ is a pre-exponential factor, $k$ is Boltzmann constant and $E_{\mathrm{a}}$ is an activation energy for oxygen ionic conduction. $E_{\mathrm{a}}$ is composed of oxygen migration enthalpy $\left(H_{\mathrm{m}}\right)$ and an enthalpy $\left(H_{\text {assoc }}\right)$ of association between oxygen vacancy and doped $\mathrm{Y}^{3+}$ ion. ${ }^{30)}$ The association between oxygen vacancy and $\mathrm{Y}^{3+}$ ion will be significant at relatively low temperatures, whereas the association becomes negligible at higher temperatures due to thermal effects. ${ }^{13,31)}$ Thus it can be considered that $E_{\mathrm{a}}$ is equal to $H_{\mathrm{m}}$ in high-temperature range, but $E_{\mathrm{a}}$ is the sum of $H_{\mathrm{m}}$ and $H_{\text {assoc }}$ in low temperature range.

In order to estimate $H_{\mathrm{m}}$ and $H_{\text {assoc }}$ values of deformed YSZ, we assumed that $H_{\mathrm{m}}$ is close to be $E_{\mathrm{a}}$ in the temperature region over $850^{\circ} \mathrm{C}$, and $H_{\text {assoc }}$ was obtained by subtracting $H_{\mathrm{m}}$ from the $E_{\mathrm{a}}$ in the temperature region below $800^{\circ} \mathrm{C}$. Table 1 shows the estimated values of $H_{\mathrm{m}}$ and $H_{\text {assoc }}$ for deformed and undeformed YSZ single crystals. According to Manning et al. ${ }^{32)} \mathrm{H}_{\mathrm{m}}$ for $9.5 \mathrm{~mol} \% \mathrm{Y}_{2} \mathrm{O}_{3}-\mathrm{ZrO}_{2}$ single crystals in the high temperature range is reported to be $81 \mathrm{~kJ} / \mathrm{mol}$. The value $(86 \mathrm{~kJ} / \mathrm{mol})$ of $H_{\mathrm{m}}$ for undeformed YSZ single crystals in this study is close to the reported value. It was found that $H_{\mathrm{m}}$ for deformed samples were smaller than that for undeformed samples, whereas $H_{\text {assoc }}$ for deformed samples were larger. Therefore, $H_{\mathrm{m}}$ and $H_{\text {assoc }}$ values were slightly changed by the plastic deformation (introduction of the dislocations in the crystal).

Table 1 The activation energy for oxygen ionic conduction $\left(E_{\mathrm{a}}\right)$, migration enthalpy $\left(H_{\mathrm{m}}\right)$ and association enthalpy $\left(H_{\mathrm{assoc}}\right)$ of the deformed and undeformed YSZ single crystals. $\Delta_{\text {deformed-undeformed }}$ represents the changes of the $E_{\mathrm{a}}, H_{\mathrm{m}}$ and $H_{\mathrm{assoc}}$ due to the plastic deformation, which was obtained by subtracting the $E_{\mathrm{a}}, H_{\mathrm{m}}$ and $H_{\text {assoc }}$ for undeformed one from the values for deformed one, respectively.

\begin{tabular}{lccc}
\hline & $H_{\mathrm{m}} / \mathrm{kJ} \cdot \mathrm{mol}^{-1}$ & $H_{\mathrm{assoc}} / \mathrm{kJ} \cdot \mathrm{mol}^{-1}$ & $\begin{array}{c}E_{\mathrm{a}}\left(H_{\mathrm{m}}+H_{\mathrm{assoc}}\right) \\
/ \mathrm{kJ} \cdot \mathrm{mol}^{-1}\end{array}$ \\
\hline $10 \%$ deformed & 79.1 & 29.0 & 108.1 \\
Undeformed & 85.9 & 21.2 & 107.1 \\
$\Delta_{\text {deformed-undeformed }}$ & -6.8 & +7.8 & +1.0 \\
\hline
\end{tabular}




\section{Discussion}

As shown above, the $1 \%$ or $10 \%$ deformed YSZ samples exhibited higher electrical conductivities than the undeformed ones. Since a number of dislocations were generated by the plastic deformation, it is thought that the introduced dislocations contribute to the increase in the ionic conductivity of YSZ. It has been well known that dislocations in metals and ceramics can act as rapid diffusion paths for vacancies and interstitials, which is called as pipe diffusion. ${ }^{19-22)}$ For example, Tang et al. reported that pipe diffusion rates of oxygen in $\alpha-\mathrm{Al}_{2} \mathrm{O}_{3}$ are by $10^{6}-10^{7}$ times larger than the bulk diffusion rates. ${ }^{22)}$ This can also confirmed from Table 1, where the $H_{\mathrm{m}}$ value of $10 \%$ deformed YSZ becomes smaller than that of undeformed YSZ.

In contrast, the $H_{\text {assoc }}$ value of $10 \%$ deformed YSZ was found to be larger as compared to that of undeformed YSZ. In undeformed YSZ, it is considered that $H_{\text {assoc }}$ comes from attractive interactions between oxygen vacancies and doped $\mathrm{Y}^{3+}$ ions. Therefore, the increase in $H_{\text {assoc }}$ of $10 \%$ deformed YSZ is due to additional interactions of oxygen vacancies with the introduced dislocations. A dislocation has an elastic stress field around it due to the lattice discontinuity at the core. Such a stress field interacts with that of point defects so as to accommodate their excess elastic energies. As a result, point defects in a crystal tend to migrate and segregate to a dislocation line, forming a so-called Cottrell atmosphere. ${ }^{33)}$ In the present case, oxygen vacancies easily migrate in the YSZ lattice, and thus oxygen-vacancy enriched regions might be formed around the dislocations. In Fig. 5, however, the ionic conductivity of the $10 \%$ deformed samples became close to these of undeformed YSZ beyond $850^{\circ} \mathrm{C}$. Therefore, in such a high-temperature range, the association of oxygen vacancies with doped $\mathrm{Y}^{3+}$ ions and dislocation become negligible, which indicates that oxygen vacancies freely diffuse through the YSZ lattice, which is similar to those in undeformed YSZ. Because dislocation structures did not change caused by heat treatments (see Fig. 3), doped $\mathrm{Y}^{3+}$ ions hardly diffused in YSZ in the temperature range below $1000^{\circ} \mathrm{C}$.

It can be seen in Fig. 5 that the ionic conductivities of deformed YSZ show different values, depending on the current direction. For both $1 \%$ and $10 \%$ deformed samples, the $\sigma_{\mathrm{n}}$ values along [ $\left.\overline{1} \overline{1} 1\right]$ were found to be larger than those along [1 $\overline{1} 0]$ over the entire temperature range. This may be related to the dislocation structures in deformed YSZ. From the TEM observations (Fig. 2), the primary slip system of (001)[110] was mainly activated at the $1 \%$ strain, relatively straight dislocation lines with $\boldsymbol{b}=a / 2[110]$ were introduced. In contrast, multiple slip systems were activated at the $10 \%$ strain, dislocation lines with various burgers vectors were more awry and tangled (Fig. 3). If the mobility of oxygen ion is sensitive to the dislocations structures, it can be considered that the difference of the electrical conductivity with respect to the current direction at the $1 \%$ strain will be larger than that at the $10 \%$ strain. In fact, when we compared the $\sigma_{\mathrm{n}}$ along [ $\overline{1} \overline{1} 1]$ with that along [1 $\overline{1} 0]$ at $650^{\circ} \mathrm{C}$ (see Fig. 5), the ratio between the $\sigma_{\mathrm{n}}$ along [ $[\overline{1} \overline{1} 1]$ and $[1 \overline{1} 0]$ at the $1 \%$ strain was 1.05 , while 1.02 at the $10 \%$ strain. Therefore, it can be said that the mobility of oxygen vacancies is dependent on the dislocation structures.

One of the possible explanations for the current direction dependence of $\sigma_{\mathrm{n}}$ in deformed YSZ is a difference in oxygen mobility with respect to the dislocation characters. As shown in Fig. 2, the introduced dislocation lines were not completely straight ever in the $1 \%$ deformed samples, and thus the dislocations have both edge and screw components. In the case of an edge dislocation, that induces hydrostatic pressures normal to the dislocation line, which will attract oxygen vacancies with the characteristic elastic field. This may contribute to association between oxygen vacancies and an edge dislocation. In contrast, a screw dislocation have shear stresses parallel to the dislocation line, and thus oxygen vacancies probably no longer have interactions with a screw dislocation, which would favor more rapid diffusion of oxygens around screw components of a dislocation. Due to the difference in strain fields from edge and screw components of dislocations, it is likely that the mobility of oxygen vacancies is affected by the dislocation characters, resulting in the current direction dependence of $\sigma_{\mathrm{n}}$ in the deformed YSZ samples. However, it is difficult to measure the oxygenion mobility along each dislocation components experimentally. In order to clarify this issue, atomistic simulations or theoretical analysis would be necessary, and yet this is beyond the scope of this study.

M'Peco et al. previously reported the ionic conductivity of the elastically deformed tetragonal zirconia polycrystals (TZP), and found that the electrical resistance of TZP increased by about $15 \%$ under the elastic stresses. ${ }^{34)}$ It should be noted here that elastically deformed TZP samples by M'Peco et al. were in the polycrystalline state, and their resistance contained components of grain boundaries as well as grain interiors. Grain boundaries in YSZ were reported to act as barriers for oxygen diffusion, and thus the resistance at the grain boundaries may become larger by the elastic stresses. In contrast, the present results indicate the increase in the ionic conductivities of YSZ crystal by plastic deformations.

Therefore, when YSZ crystals are more deformed and the dislocation density is increased, it can be expected to attain further enhancement of the ionic conductivities in YSZ. This would be a novel technique to improve the electrical properties of $\mathrm{ZrO}_{2}$-related ceramics.

\section{Conclusions}

YSZ single crystals were plastically deformed by hightemperature compression tests. Subsequently, we observed the dislocation structures and distribution, and measured electrical conductivity of deformed YSZ single crystals. The results obtained in this study are summarized as follow.

(1) Plastic deformation enhanced electrical conductivities of YSZ single crystals, independent of the crystallographic orientation.

(2) The electrical conductivity for deformed YSZ single crystals increased with increasing temperature in the temperature range of $250-800^{\circ} \mathrm{C}$.

(3) The $\sigma_{\mathrm{n}}$ for deformed YSZ single crystals decreased to the value for undeformed YSZ single crystals in the temperature range of $850-1000^{\circ} \mathrm{C}$. 
(4) Since the migration enthalpy of oxygen vacancies decreased and the association enthalpy increased by plastic deformation, oxygen vacancies concentrated around the dislocations may move very quickly along the dislocation lines.

\section{Acknowledgements}

This work was supported by PRESTO, Japan Science and Technology Agency. A part of this work was financially supported by a Grant-in-Aid for Japan Society for the promotion of Science Fellows (14-08214) from the Ministry of Education, Culture, Sports, Science and Technology, Japan.

\section{REFERENCES}

1) R. J. Ackermann, E. D. Rauh and C. A. Alexander: High Temp. Sci. 7 (1975) 304-316.

2) D. Viechnicki and V. S. Stubican: J. Am. Ceram. Soc. 48 (1965) $292-$ 297.

3) E. C. Subbarao, H. S. Maiti and K. K. Srivastava: Phys. Status Solidi A. 21 (1975) 9-40.

4) C. F. Grain: J. Am. Ceram. Soc. 50 (1967) 288-296.

5) H. G. Scott: J. Mater. Sci. 10 (1975) 1527-1535.

6) H. M. Ondik and H. F. McMurdie: Phase diagrams for zirconium and zirconia systems, (The American Ceramic Society, Westerville, Ohio, 2002) pp. 35-343.

7) N. Shibata, J. Katamura, A. Kuwabara, Y. Ikuhara and T. Sakuma: Mater. Sci. and Eng. A. 312 (2001) 90-98.

8) J. Katamura, N. Shibata, Y. Ikuhara and T. Sakuma: Philos. Mag. Lett. 78 (1998) 45-49.

9) H. Ogawa, A. Yasuda, N. Shibata, Y. Ikuhara and T. Sakuma: Philos. Mag. Lett. 77 (1998) 199-203.

10) A. Kuwabara, S. Yokota, Y. Ikuhara and T. Sakuma: Mater. Sci. Forum. 357-359 (2001) 399-404.

11) J. A. Kiliner and C. H. Steele: Nonstoichiometric oxide, ed. by O. Toft Sø rensen, (Academic Press, New York, 1981) pp. 263-299.

12) N. Q. Minh: J. Am. Ceram. Soc. 76 (1993) 563-588.
13) Y. Arachi, H. Sakai, O. Yamamoto, Y. Takada and N. Imanishai: Solid State Ionics 121 (1999) 133-139.

14) M. Aoki, Y. M. Chiang, I. Kosacki, L. J.-R. Lee, H. Tuller and Y. Liu: J. Am. Ceram. Soc. 79 (1996) 1169-1180.

15) M. J. Verkerk, B. J. Middelhuis and A. J. Burggraaf: Solid State Ionics. 6 (1982) 159-170.

16) I. Kosacki, T. Suzuki, V. Petrosky and H. U. Anderson: Solid State Ionics 136-137 (2000) 1225-1233.

17) H. L. Tuller: Solid State Ionics 131 (2000) 143-157.

18) A. Atkinson and C. Monty: Surfaces and Interfaces of Ceramic Materials, ed. by L. C. Dufour et al. (Kluwer Academic, Dordrecht, 1989) pp. 273.

19) J. P. Hirth and J. Lothe: Theory of dislocations, 2nd ed. (Wiley, McGraw-Hill, New York, 1982) pp. 497-506.

20) R. W. Balluffi and A. V. Granato: in Dislocations in solids, Vol. 4 Chap. 13. ed. by F. R. N. Nabarrro, (North-Holland, Amsterdam, 1979). pp. 23-31.

21) A. Nakamura, K. Matsunaga, J. Tohma, T. Yamamoto and Y. Ikuhara: Nature Materials 2 (2003) 453-456.

22) X. Tang, K. P. D. Lagerlöf and A. H. Heuer: J. Am. Ceram. Soc. 86 (2003) 560-565

23) K. Otsuka, A. Kuwabara, A. Nakamura, K. Matsunaga, T. Yamamoto and Y. Ikuhara: Appl. Phys. Lett. 82 (2003) 877-879.

24) A. Dominguez-Rodriguez, V. Lanteri and A. H. Heuer: J. Am. Ceram. Soc. 69 (1986) 285-287.

25) E. Fries, F. Guiberteau, A. Dominguez-Rodriguez, D. S. Cheong and A. H. Heuer: Philos. Mag. A. 60 (1989) 107-121.

26) D. S. Cheong, A. Dominguez-Rodriguez and A. H. Heuer: Philos. Mag. A. 60 (1989) 123-138.

27) D. S. Cheong, A. Dominguez-Rodriguez and A. H. Heuer: Philos. Mag. A. 63 (1991) 377-388

28) J. R. MacDonald: Impedance Spectroscopy: Emphasizing Solid Materials and Systems, (Wiley, New York, 1987) pp. 191-260.

29) J. E. Bauerle: J. Phys. Chem. Solids. 30 (1969) 2657-2670.

30) J. A. Kilner and R. J. Brook: Solid State Ionics. 6 (1982) 237-252.

31) J. A. Kilner and C. D. Waters: Solid State Ionics. 6 (1982) 253-259.

32) P. S. Manning, J. D. Sirman, R. A. De Souza and J. A. Kilner: Solid State Ionics 100 (1997) 1-10.

33) A. H. Cottrell and M. A. Jaswon: Proc. R. Soc. Lond. A 199 (1949) 104-114.

34) J.-C. M'Peko, D. L. S. Jr and M. F. de Souza: Appl. Phys. Lett. 81 (2002) 2827-2829. 